\title{
Improved Video Segmentation by Adaptive Combination of Depth Keying and Mixture-of-Gaussians
}

\author{
Ingo Schiller and Reinhard Koch \\ Multimedia Information Processing (MIP) \\ Institute of Computer Science, University of Kiel, Germany \\ \{ischiller, rk\}@mip.informatik.uni-kiel.de \\ http://www.mip.informatik.uni-kiel.de
}

\begin{abstract}
Video segmentation or matting, the separation of foreground objects from background in video sequences, is a demanding task and is needed for a broad range of applications. The most widespread method for video segmentation is chroma-keying using a known background color for which a controlled environment is required. Recently a different method of keying fore-and background has been proposed in which the chroma-keying is replaced by depth-keying using a Time-of-Flight (ToF) camera. The current ToF-cameras suffer from noise and low resolution sensors, which results in unsatisfying segmentation results. We propose to combine the segmentation of dynamic objects in depth with a segmentation in the color domain using adaptive background models. We weight the two measures depending on the actual depth values using either the variance of the depth images of the ToF-camera or the amplitude image of the ToF-camera as reliability measure. We show that both methods significantly improve the segmentation results.
\end{abstract}

\section{Introduction}

Today, television content as well as movie content contains a significant amount of artificially composed scenes. To generate such scenes with a mixture of real and artificial content the real foreground objects have to be separated from the background. The most common and reliable method for this segmentation task is to use chroma-keying techniques with a known background color as described in [6]. Wang and Cohen [8] give a very good overview of the field of matting and segmentation for still images and extend the discussion to video streams. In their survey they discuss many different approaches such as Poisson Matting, Graph Cut Matting, Baysian Matting, Random Walk matting and others. A combined segmentation approach based on depth from stereo and color using Mixture-of-Gaussians was presented by Gordon et al. in [4], in which all pixels detected by either color or depth are considered foreground. The usage of active distance measurement devices providing interactive frame-rates for segmentation has first been introduced by Gvili et al. in [5] and was extended in [1] to segment dynamic objects with a Time-of-Flight (ToF)-camera [10]. Crabb et al. [2] propose the generation of a Trimap from ToF-depth and use Cross-Bilateral filtering for segmentation. They

A. Heyden and F. Kahl (Eds.): SCIA 2011, LNCS 6688, pp. 59-68,2011.

(C) Springer-Verlag Berlin Heidelberg 2011 
however miss to show results of more challenging scenes so that a real evaluation is not possible. Zhu et al. [11] use a combination of stereo and ToF-camera for video matting which shows promising results. The complexity of the approach however does not allow real-time usage. Gong et. al [3] very recently extended the Poisson Matting approach for the usage of additional ToF-depth images using Trimaps. In contrast to their work and most other recently proposed methods, no Trimaps are required in our proposed segmentation algorithm.

The use of a ToF-camera that delivers depth for each pixel facilitates object segmentation, because depth is invariant to changing scene illumination and object shadows. However, ToF depth images typically have low resolution compared to color CCD images, hence a combination of depth and color segmentation is desirable. In our system we combine a ToF-camera $(204 \times 204$ pixel $)$ with a color CCD-camera $(1600 \times 1200$ pixel) (see figure 1 (a)) by warping the depth data into the CCD video stream using the approach described in [1]. We propose to combine segmentation of depth measurements by a ToF-camera with the foreground detection by Mixture-of-Gaussians (MoG). We adaptively weight the two modalities dependent on the current reliability of the depth measurement. To determine the reliability of depth measurements we compare the usage of the depth variance and amplitude image. We argue that the two methods mutually improve the deficits of the other and with the proposed weighting it is possible to significantly improve the segmentation results.

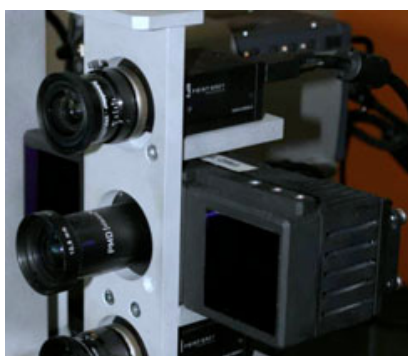

(a)

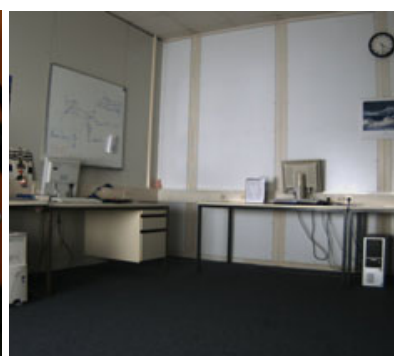

(b)

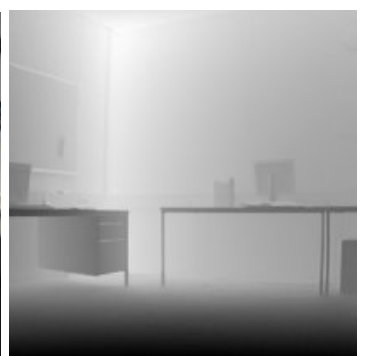

(c)

Fig. 1. The capturing system (a) with a CCD camera above a PMDTec CamCube ToF-camera. Averaged background CCD- (b) and depth- image (c). Brighter values indicate bigger distances.

\section{Segmentation}

In this section we discuss the segmentation of foreground objects using depth thresholding and the Mixture-of-Gaussians method which we also extend to a forth depth channel. Finally we introduce our proposed method which adaptively weights color and depth clues.

\subsection{Segmentation by Depth Keying}

The most obvious way to detect moving objects is to compare a background depth image of the scene and the current depth image delivered by the ToF-camera. The 
background depth can be created by averaging several ToF-images (see figure 1). In the segmentation phase the depth $z(x)$ of every pixel $x$ of a ToF-image is compared to the background image pixel's depth $z_{b}(x)$ and if: $z(x)<z_{b}(x)-\tau$ the pixel $x$ is classified as foreground or moving object ( $\tau$ is a threshold).

The keying is executed on the GPU using shaders in the domain of the CCD-camera. Figure 2 shows the depth- (a) and corresponding CCD-image (b) with a person in the scene. Image (c) shows the keying result on the depth image and (d) shows the corresponding color result. This simple keying approach has one main limitation: the segmentation is not very precise at object boundaries due to noise and the low depth resolution. Therefore an improved segmentation at object boundaries is needed.

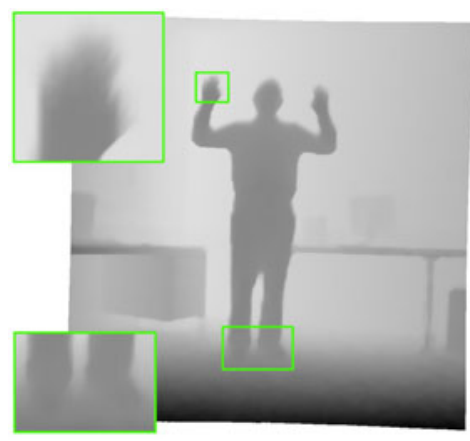

(a)

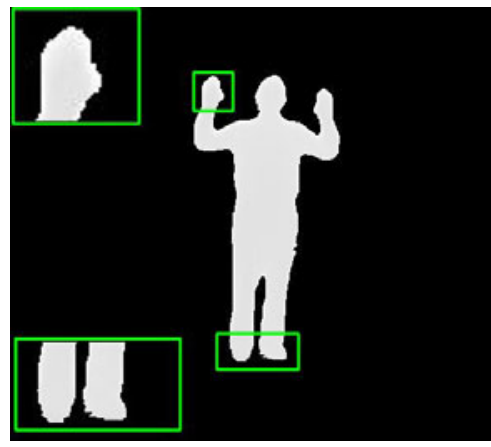

(c)

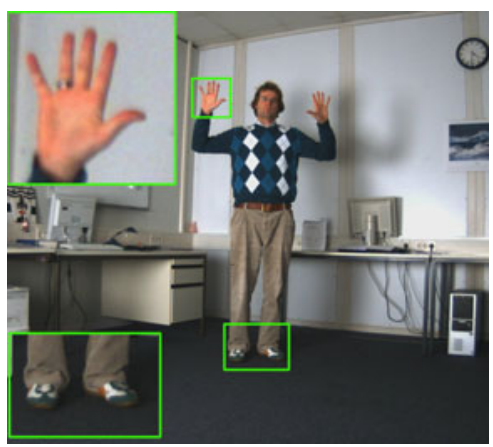

(b)

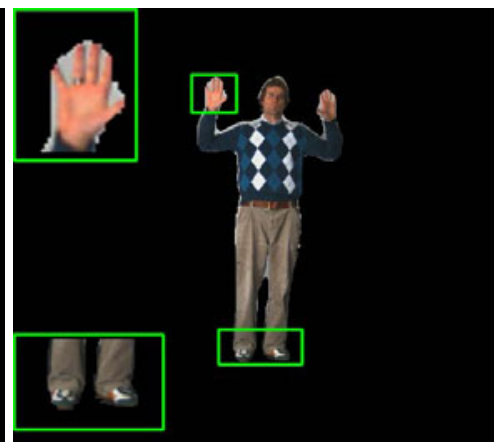

(d)

Fig. 2. Warped depth image with person (a), Corresponding CCD image (b), depth segmented image (c) and corresponding CCD image (d)

\subsection{Segmentation by Mixture-of-Gaussians}

A well-known method for segmentation is Mixture-of-Gaussians (MoG). The detection of dynamic objects in a scene using an adaptive background mixture model relates to the work of $\mathrm{Xu}$ et al. [9] and Stauffer et al. [7]. They describe a method that uses multiple Gaussian distributions to model each pixel of an image. An intensity image is defined by the three color channels in RGB space $F=\left(F_{R}, F_{G}, F_{B}\right)$. Assuming 
that no channel is saturated this representation can be transformed into a normalized form $f=\left(f_{r}, f_{g}, f_{b}\right)$ with e.g. : $f_{r}=F_{R} / \sqrt{F_{R}^{2}+F_{G}^{2}+F_{B}^{2}}$. It is concluded that it is appropriate to model each $f_{k} \in f$ using a Gaussian distribution and the probability of observing a value $f_{t}=\left(f_{r, t}, f_{g, t}, f_{b, t}\right)$ at a pixel at time $t$ is therefore given by:

$$
P\left(f_{t}\right)=\sum_{i=1}^{N} \omega_{i, t} \frac{1}{\sqrt{2 \pi} \sigma_{i, t}} e^{-\frac{\left(f_{t}-\mu_{i, t}\right)^{2}}{2 \sigma_{i, t}^{2}}}
$$

where $N$ is the number of distributions, $\omega_{i, t}$ is a weighting factor for each distribution, $\sigma_{i, t}^{2}$ are the variances and $\mu_{i, t}$ the mean of the Gaussian distributions. Initial values for variance $\sigma_{0,0}^{2}$ and mean $\mu_{0,0}$ are determined over a small image region $\Delta x$. A pixel is classified as belonging to a distribution $i$ if:

$$
\left\|f_{t}-\mu_{i, t-1}\right\|<c \sigma_{i, t-1},(c \approx 3)
$$

and the parameters are updated accordingly. The weights $\omega_{i, t}(x)$ are increased while the weights of the not matched distributions $\omega_{j, t}(x)$ are decreased. If the current pixel does not match any of the existing distributions a new distribution is generated, and if the number of distributions exceeds a maximum (we allow 3 background distributions), the distribution with the lowest weight is deleted. In our implementation we record about 50 images of the empty scene and build the background distributions. After that the update of the background distributions is disabled and only the distances (see equation (2)) to the background distributions are computed. The average distance of a pixel $x$ to the background distributions is the color weight and denoted $c(x)$ in the following.

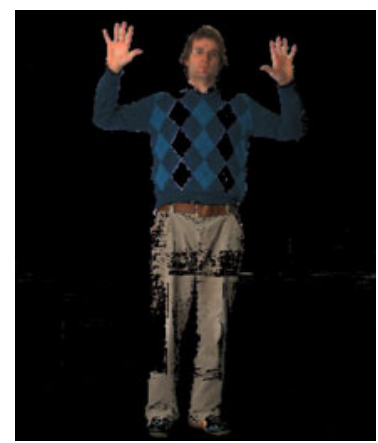

(a) Depth

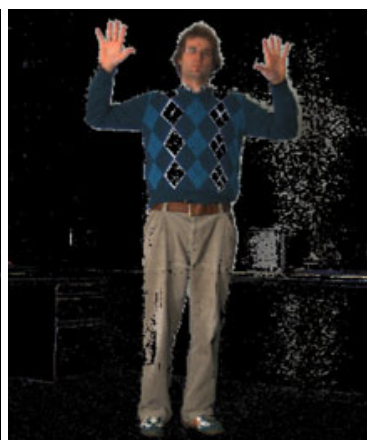

(b) MoG (rgb)

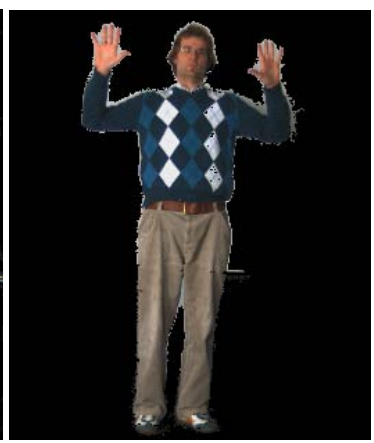

(c) MoG+Depth (rgbd)

Fig. 3. MoG segmentation result: The color segmentation easily under- or over-segments foreground objects (a), (b). MoG with depth as fourth channel improves the result, but borders remain erroneous (c).

Figure 3 (a) and (b) shows a segmentation result if only the Mixture-of-Gaussian on color is used as segmentation clue. It can be seen that the algorithm tends to either under- or over-segment the person, especially if a challenging scene is chosen with many shadows and colors similar to the background. 


\subsection{Combining Color and Depth}

From the results of the depth- and MoG- segmentation we conclude that a combination of the two approaches will improve the segmentation. The crucial decision is, which segmentation is more likely to be correct and if the two approaches deliver different results which one to trust. The first intuitive possibility is to add the depth information as forth channel to the MoG on color images. The vector on which the distributions are defined is then formed by the three color channels in rgb space and the depth value $d$ as combined rgbd space: $f=\left(f_{r}, f_{g}, f_{b}, d\right)$. Figure 3(c) shows that this can compensate some of the shortcomings of the pure MoG segmentation, but the borders of the foreground person are still erroneous.

Therefore we propose to use a reliability measure for depth information in the segmentation. We evaluate two different approaches as reliability measure. The first (method A) is the usage of the variance in depth and the second (method B) is the usage of the amplitude information provided by the ToF-camera. The amplitude image quantifies the amount of light that is reflected from the object to the camera. The higher the values the more reliable is the measurement. At object discontinuities less light is reflected due to scattering effects. Therefore we use the inverse of the amplitude image to enable its usage in the same way as the variance image.

Discontinuities in method A are detected by analyzing the variance in the original depth image. High variances are marked as shown in figure 4 (a). To be able to compare the different modalities they have to be normalized. The current depth difference $d(x)=z(x)-z_{b}(x)$ is normalized between the minimum $d_{\min }$ and the maximum depth difference $d_{\max }$ in that image and in the same way the weight of the color foreground pixels $c(x)$ is normalized between the minimum and maximum color weights $c_{\min }$ and $c_{\max }$ :

$$
\hat{d}(x)=\frac{d(x)-d_{\min }}{d_{\max }-d_{\min }} \quad \hat{c}(x)=\frac{c(x)-c_{\min }}{c_{\max }-c_{\min }}
$$

The variance and inverted amplitude values are also normalized between zero and one to be comparable to the other measurement weights $\hat{d}(x)$ and $\hat{c}(x)$, and denoted the normalized uncertainty $\hat{v}(x)$. In areas in which the depth uncertainty is high, the depth measurement is considered unreliable. Therefore we weight the normalized depth difference $\hat{d}(x)$ with the uncertainty $\hat{v}(x)$, resulting in an uncertainty filtered depth $\hat{d v}(x)$ which is scaled between zero and one dependent on the uncertainty. In contrast to that is the color more reliable if the depth uncertainty is high. Therefore the color weight $\hat{c}(x)$ is multiplied with the depth uncertainty $\hat{v}(x)$ and added to the color weight. The result is that if the depth uncertainty is high the color weight is weighted even higher while at the same time the uncertainty filtered depth is weighted lower. To consider all measures in an adequate manner the following equations are proposed:

$$
\begin{array}{r}
\hat{d v}(x)=(1-\hat{v}(x)) \hat{d}(x) \\
\hat{c} v(x)=(1+\hat{v}(x)) \hat{c}(x) \\
\hat{s}(x)=\frac{1}{2}(\hat{d v}(x)+\hat{c} \hat{v}(x))
\end{array}
$$




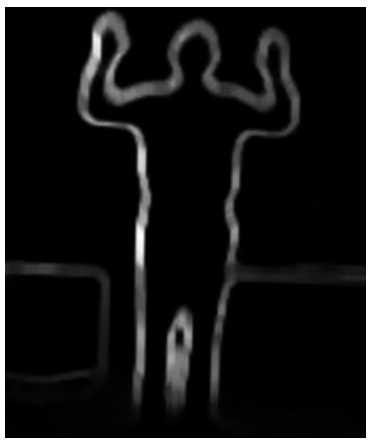

(a)

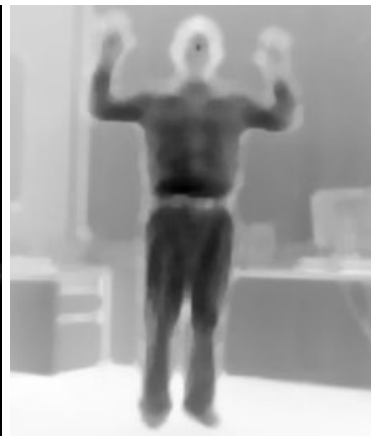

(b)

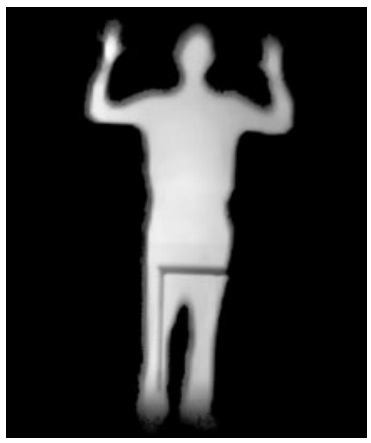

(c)

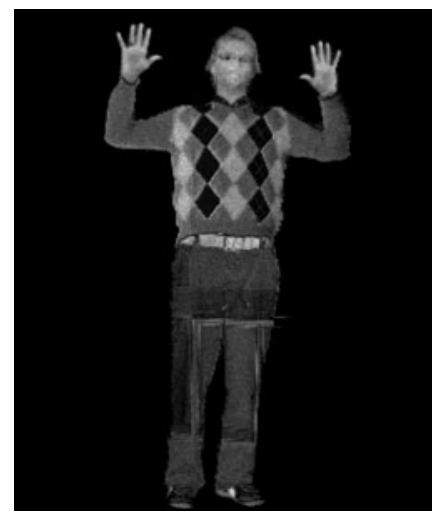

(d)

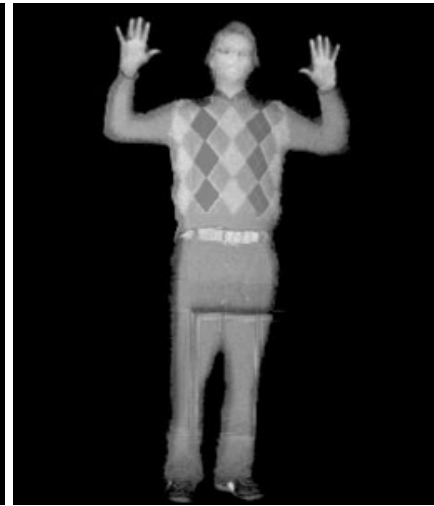

(e)

Fig. 4. Weighting images:(a)+(b) variance weight image and amplitude image (uncertainty) $\hat{v}(x)$, (c) variance weighted depth difference $\hat{d v}(x)$, (d) MoG weight $\hat{c}(x)$ and (e) combined weight image $\hat{s}(x)$

Figure 4 shows the weighting images of the proposed approaches. Image (b) shows the inverted normalized amplitude image, (c) the normalized variance weighted depth image, (d) the weighting image of the MoG and the combined weighting image $\hat{s}(x)$. Brighter values indicate higher weights. It is clearly visible that the color segmentation gains more importance on fine structures such as the hands and the feet of the person. For finally composing the image $C$ (see images in figure 7), we use blending between foreground $F$ and background color $B$ with $\hat{s}(x)$ the matting alpha:

$$
C=\hat{s}(x) F+(1-\hat{s}(x)) B
$$

\section{Results}

The approaches are evaluated with the depth maps warped to the domain of the color camera. Two images have been labeled by hand (see figure 5(a) and (b)) to allow quantitative evaluation. We chose challenging examples in which colors of the background 


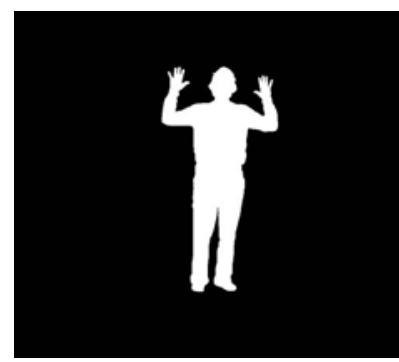

(a)

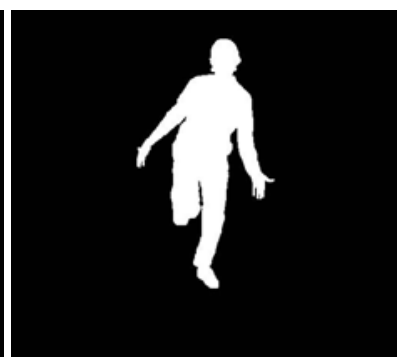

(b)

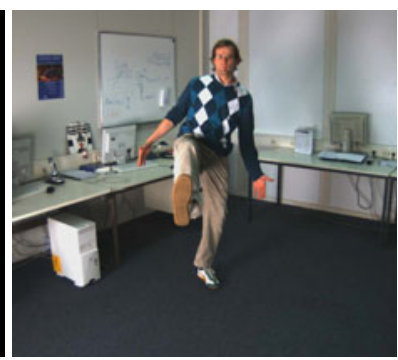

(c)

Fig. 5. Manually labeled silhouette images for quantitative evaluation in tables 1 (a) and2(b). (c) shows the to (b) corresponding color image and (a) corresponds to the images in figure 2

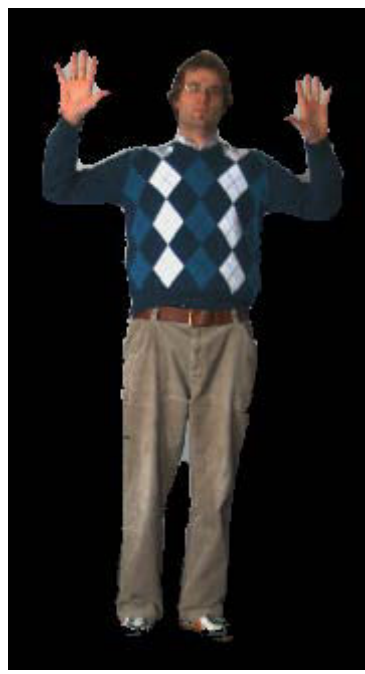

(a) MoG with variance (b) weighted depth

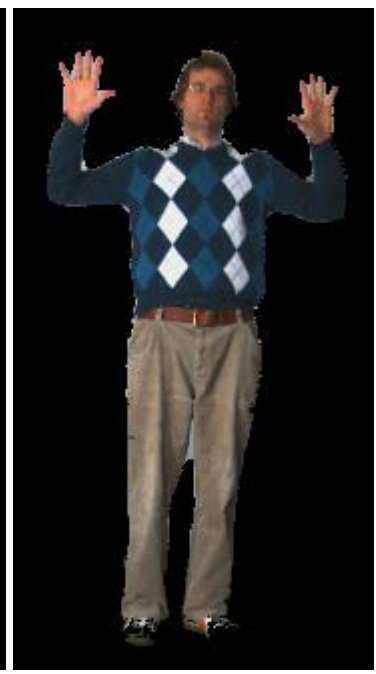

(b) MoG with amplitude weighted depth

Fig. 6. Segmented images, corresponding to table1 (a) Proposed method A, (b) proposed method B. Note the increased segmentation quality at the borders, especially at the top of the person.

are also present in the foreground and in which we try to distinguish a person from the floor it is standing on. Tables 1 and 2 show the evaluation results of the segmentation for the different approaches. The tables compare the number (\#) of matching pixel, which describes how many pixel have been correctly identified as fore-or background, how many false positives (detected as foreground, but belonging to background) and how many false negatives are produced by the different approaches. The computed matte values $\hat{s}(x)$ have been thresholded for this purpose to obtain a binary segmentation result with $\hat{s}(x)>0.1$. Percentages of matching pixel are relative to the number of pixel, percentages of false positives, false negatives and the total error are given relative to the number of foreground pixel. 
Table 1. Segmentation evaluation for image (a) of figure 5 The image consists of 225000 pixel of which 18087 have been manually selected as foreground.

\begin{tabular}{c|c|c|c|c} 
Approach & $\begin{array}{c}\text { Matching } \\
\text { pixel \#/\% }\end{array}$ & $\begin{array}{c}\text { False } \\
\text { positives \#/\% }\end{array}$ & $\begin{array}{c}\text { False } \\
\text { negatives \#/\% }\end{array}$ & $\begin{array}{c}\text { Total error } \\
\# / \%\end{array}$ \\
\hline Depth & $223125 / 99.167$ & $1542 / 8.287$ & $333 / 1.79$ & $1875 / 10.076$ \\
MoG & $221207 / 98.314$ & $2320 / 12.468$ & $1473 / 7.916$ & $3793 / 20.384$ \\
MoG+Depth & $222098 / 98.710$ & $2680 / 14.402$ & $222 / 1.193$ & $2902 / 15.595$ \\
Proposed Method A & $223782 / 99.459$ & $599 / 3.219$ & $619 / 3.327$ & $1218 / 6.546$ \\
Proposed Method B & $223782 / 99.471$ & $561 / 3.015$ & $630 / 3.386$ & $1191 / 6.4$
\end{tabular}

Figure 7 shows the segmentation results of the combined approach. At some particular difficult points, in this example the shoes of the person, the segmentation is not entirely correct, because the similarity between the white shoes and the gray floor is too high after color normalization. The improved segmentation is clearly visible at hands,

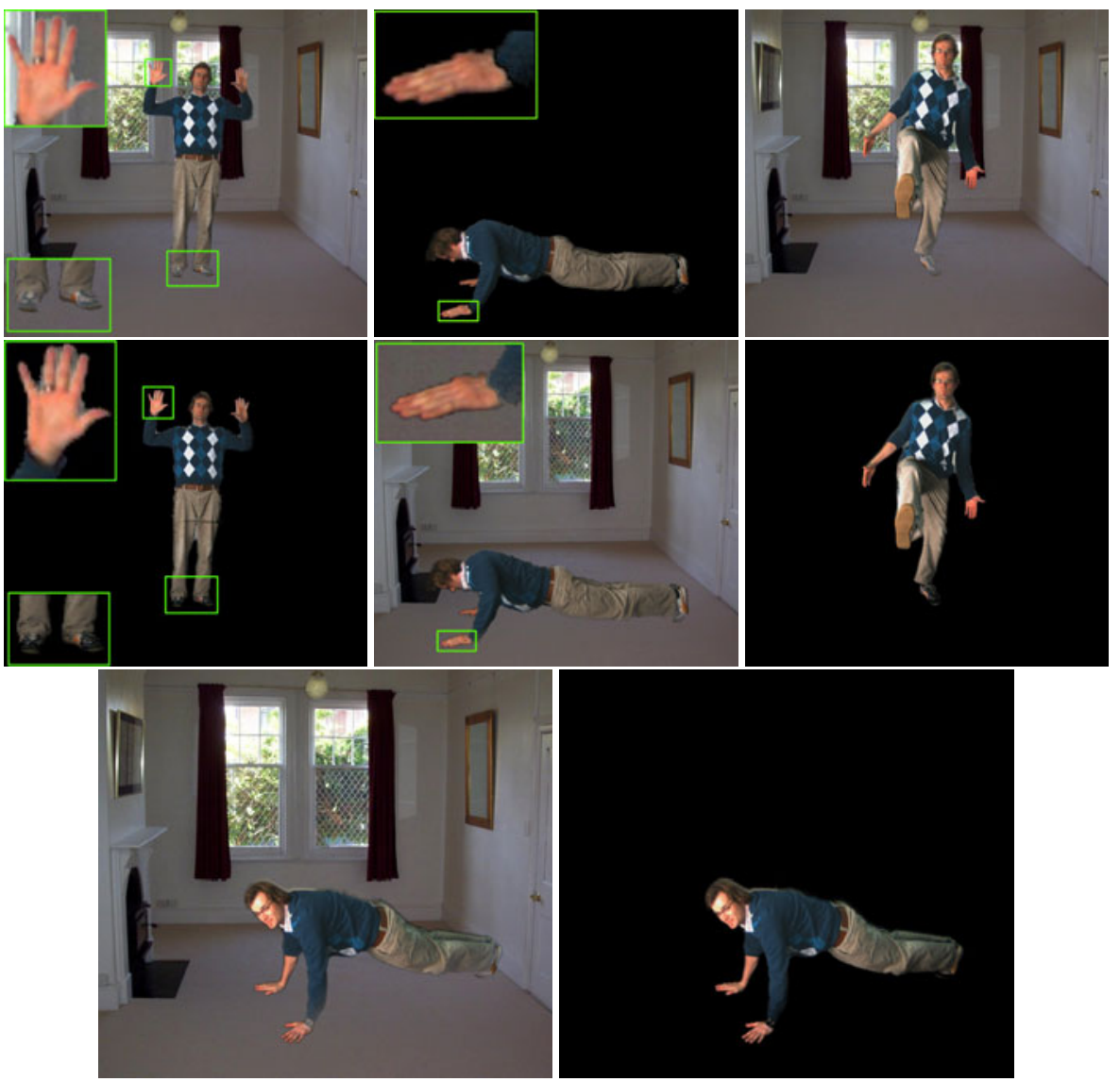

Fig. 7. Final results blended with background image and with black background using equation 7 Some of the improved regions are marked and enlarged. 
Table 2. Segmentation evaluation for image (b) of figure 5 The image consists of 225000 pixel of which 20859 have been manually selected as foreground.

\begin{tabular}{c|c|c|c|c} 
Approach & $\begin{array}{c}\text { Matching } \\
\text { pixel \#/\% }\end{array}$ & $\begin{array}{c}\text { False } \\
\text { positives \#/\% }\end{array}$ & $\begin{array}{c}\text { False } \\
\text { negatives \#/\% }\end{array}$ & $\begin{array}{c}\text { Total error } \\
\# / \%\end{array}$ \\
\hline Depth & $221054 / 98.246$ & $3512 / 16.837$ & $434 / 2.081$ & $3946 / 18.918$ \\
MoG & $221128 / 98.280$ & $1944 / 9.320$ & $1928 / 9.243$ & $3872 / 18.563$ \\
MoG+Depth & $221522 / 98.454$ & $3300 / 15.821$ & $178 / 0.853$ & $3478 / 16.674$ \\
Proposed Method A & $222704 / 98.980$ & $1955 / 9.372$ & $341 / 1.635$ & $2296 / 11.007$ \\
Proposed Method B B & $223297 / 99.243$ & $1375 / 6.592$ & $328 / 1.572$ & $1703 / 8.164$
\end{tabular}

hair, the silhouette and feet of the person. Tables 1 and 2 show a quantitative evaluation of the different approaches. It can be clearly seen that our proposed method outperforms the other methods. The proposed method B, which utilizes the amplitude images of the ToF-camera as reliability measure, performs better than the proposed method A.

Our current implementation is not optimized for speed, but to quantify the possibilities we will give some numbers of the current implementation. The current algorithm operates at $7 \mathrm{~Hz}$ on a standard Intel Core i7 PC. Warping the depth to the CCD image takes $\approx 20 \mathrm{~ms}$, applying MoG to a color image takes $\approx 140 \mathrm{~ms}$ and segmenting the image on the GPU takes $\approx 20 \mathrm{~ms}$ including uploading the images to textures and readout of textures to images. At the moment we use two shader passes which can be reduced to one. MoG, the limiting factor, is currently executed on the CPU, parallel to the final segmentation. Transferring it to the GPU will significantly speed up the process.

\section{Conclusions}

We proposed a combined color and depth segmentation approach using a ToF-camera for depth- and Gaussian-Mixture-Models for color segmentation. Our contribution is the combination of the two approaches using the amplitude image of the ToF-camera or the depth variance as reliability measure for the depth measurements which significantly reduces segmentation errors on fine structures and image areas in which foreand background meet. The approach is real-time capable as the depth segmentation and the evaluation of the combined weighting function is processed on the GPU. The refined segmentation requires, unlike other methods, no user interaction such as the selection of a coarse outlining of the foreground object. With these qualities the approach is wellsuited for a variety of applications such as Mixed Reality approaches, teleconferencing systems or virtual studios.

\section{Acknowledgment}

This work has partially been funded by the "Zukunftsprogramm Schleswig-Holstein (2007-2013)" with funds from the European Commission (EFRE) and Land SchleswigHolstein, Germany, as part of the Initiative KoSSE, project 122-09-048. 


\section{References}

1. Bartczak, B., Schiller, I., Beder, C., Koch, R.: Integration of a time-of-flight camera into a mixed reality system for handling dynamic scenes, moving viewpoints and occlusions in real-time. In: Proceedings of the 3DPVT Workshop, Atlanta, GA, USA (June 2008)

2. Crabb, R., Tracey, C., Puranik, A., Davis, J.: Real-time foreground segmentation via range and color imaging. In: IEEE Computer Society Conference on Computer Vision and Pattern Recognition Workshops (CVPRW), pp. 1-5 (June 2008)

3. Gong, M., Wang, L., Yang, R., Yang, Y.H.: Real-time video matting using multichannel poisson equations. In: Proceedings of Graphics Interface (GI), pp. 89-96. Canadian Information Processing Society, Toronto (2010), http: //portal.acm.org/citation.cfm?id=1839214.1839231

4. Gordon, G., Darrell, T., Harville, M., Woodfill, J.: Background estimation and removal based on range and color. In: IEEE Computer Society Conference on Computer Vision and Pattern Recognition (CVPR), vol. 2, pp. 458-464 (1999)

5. Gvili, R., Kaplan, A., Ofek, E., Yahav, G.: Depth keying, vol. 5006, pp. 564-574. SPIE (2003), http://link.aip.org/link/?PSI/5006/564/1

6. Smith, A.R., Blinn, J.F.: Blue screen matting. In: SIGGRAPH 1996: Proceedings of the 23rd Annual Conference on Computer Graphics and Interactive Techniques, pp. 259-268. ACM, New York (1996)

7. Stauffer, C., Eric, W., Grimson, L.: Adaptive background mixture models for real-time tracking. In: IEEE Computer Society Conference on Computer Vision and Pattern Recognition (CVPR), pp. 2246-2252 (1999)

8. Wang, J., Cohen, M.F.: Image and video matting: a survey. Foundations and Trends in Computer Graphics and Vision 3(2), 97-175 (2007)

9. $\mathrm{Xu}, \mathrm{M}$., Ellis, T.: Illumination-invariant motion detection using colour mixture models. In: British Machine Vision Conference (BMVC), pp. 163-172 (2001)

10. Xu, Z., Schwarte, R., Heinol, H., Buxbaum, B., Ringbeck, T.: Smart pixel - photonic mixer device (PMD). In: International Conference on Mechatronics and Machine Vision in Practice (M2VIP), Nanjing, p. 259-264 (1998)

11. Zhu, J., Liao, M., Yang, R., Pan, Z.: Joint depth and alpha matte optimization via fusion of stereo and time-of-flight sensor. In: IEEE Computer Society Conference on Computer Vision and Pattern Recognition (CVPR), pp. 453-460. IEEE, Los Alamitos (2009) 